\title{
Attributable Costs of Postoperative Atrial Fibrillation among Patients Undergoing Cardiac Surgery
}

\author{
Pattamawan Kosuma, ${ }^{1}$ Sitichok Wachirasrisirikul, ${ }^{2}$ and Arom Jedsadayanmata $\mathbb{1 D}^{3}$ \\ ${ }^{1}$ Department of Pharmacy Practice, Naresuan University, Phitsanulok, Thailand \\ ${ }^{2}$ Department of Surgery, Buddhachinaraj Hospital, Phitsanulok, Thailand \\ ${ }^{3}$ Faculty of Pharmacy, Thammasat University, Pathum Thani, Thailand \\ Correspondence should be addressed to Arom Jedsadayanmata; aromj@tu.ac.th
}

Received 7 December 2017; Accepted 13 June 2018; Published 28 June 2018

Academic Editor: Mariantonietta Cicoira

Copyright ( 2018 Pattamawan Kosuma et al. This is an open access article distributed under the Creative Commons Attribution License, which permits unrestricted use, distribution, and reproduction in any medium, provided the original work is properly cited.

\begin{abstract}
Background. Postoperative atrial fibrillation (POAF) is the most common complication among patients undergoing cardiac surgery. However, data on the economic burden and resource utilization associated with POAF in Asian population are limited. The present study aimed at estimating medical costs attributable to POAF after cardiac surgery in Thai population. Methods. We analysed data from claims database of patients who underwent valve replacement, coronary artery bypass grafting (CABG), or a combination of both procedures at a tertiary-care, academic hospital in Thailand. Multiple linear regressions of log-transformed costs were developed with the occurrence of POAF and preoperative patient characteristics as covariates. After back-transformation to the original scale, costs attributable to POAF were estimated from the mean difference between patients with and without POAF. Results. Of 711 patients undergoing cardiac surgery, 241 (30.94\%) developed POAF over a median hospitalization of 10 days. Patients with POAF utilized more resources than those without POAF. POAF was an independent predictor and increased cost by $23 \%$ in linear regression model. On average, patients with POAF had higher medical costs than those without POAF (269,000 versus 218,999 Thai Baht (THB)) with a mean difference of 50,000 THB (1,667 USD). The difference was observed in patients undergoing isolated valve surgery (47,761 THB (1,592 USD), 95\% CI: 39,809-55,712), CABG (50,865 THB (1,696 USD), 95\% CI: 37,233-64,496), and a combination of both procedures (72,287 THB (2,410 USD), 95\% CI: 49,910-94,405). Conclusions. In a single-institution study in Thailand, POAF is associated with increased resource use and medical costs among patients undergoing cardiac surgery. Effective strategies to prevent POAF should be implemented to reduce its economic burden.
\end{abstract}

\section{Introduction}

Postoperative atrial fibrillation (POAF) is the most common complication among patients undergoing cardiac surgery [1]. The incidences of POAF have been reported from 10 to $60 \%$ depending on the study population and POAF definition $[2,3]$. POAF usually occurs within the first 2-3 days after surgery and is associated with other postoperative complications including ischemic stroke, heart failure, and acute kidney injury $[2,3]$. Standard therapy for POAF, namely, antiarrhythmic agents and anticoagulants, could cause unwanted effects by inducing proarrhythmia and bleeding [3]. As a result, POAF may complicate the course of hospitalization, compromise clinical outcomes, prolong hospitalization, and increase costs of care among cardiac surgery patients [4-9].

Despite its potential impact on morbidity and resource utilization, few studies evaluated the economic burden of POAF in Asian population. Previous reports were conducted mostly in Western countries [4-10]. Such economic information might not be easily transferable across countries because the cost of care and resource utilization could vary among regions, depending on practice pattern and resource available. Knowing the economic burden of POAF is important to determine the impact of interventions to prevent this complication in the context of Asian health services. Thus, the present study aimed at estimating direct medical costs attributable to POAF in Thai population undergoing 
cardiac surgery. The findings will have significant implications for policy making.

\section{Methods}

2.1. Data Collection. We retrospectively analysed a cohort of patients who underwent valve replacement or coronary artery bypass grafting (CABG) or a combination of both procedures at a 1000-bed, tertiary-care, academic hospital in Thailand. All data were retrieved electronically from claims database at the study hospital. The claims database provided information on patient demographics, a principle diagnosis, types of surgery performed, comorbid conditions prior to admission, complications during hospitalization, discharge disposition, individual service charges, and pharmacy product utilization.

Patients were eligible for recruitment if they were 18 years of age or older, had undergone CABG identified with the International Classification of Diseases, Ninth Revision (ICD-9) coding system (ICD-9: 36.10 to 36.16), and/or had undergone valve surgery (ICD-9: 35.20 to 35.28 ) between January 1, 2005, and June 30, 2012. Patients were excluded if they died within 24 hours of surgery.

The occurrence of POAF was identified with the International Classification of Diseases, Tenth Revision (ICD10) code for AF (I48) when it was recorded as a complication during hospitalization. Because AF occurring prior to admission will be recorded under comorbid conditions, this enabled us to distinguish POAF from AF occurring prior to cardiac surgery.

The claims database provided data on inpatient charges from individual services during hospitalization. A unique patient identifier was used to combine datasets, enabling estimation of total charges for each patient. Charges were converted to costs with cost-to-charge ratios reported specifically in each year by the hospital accounting department. We did not adjust medical costs for inflation over the study period.

2.2. Data Analysis. Baseline characteristics between patients with and without POAF were compared using chi-square for categorical variables and the independent $t$-test or MannWhitney $U$ test as appropriate for numerical variables.

The total medical costs in Thai Baht (THB) were separated into cost components according to the services provided including supplies and devices; room, board, and nursing; pharmacy products; laboratory and testing; operations; and other services. We compared these unadjusted cost components between patients with and without POAF using mean difference and independent $t$-test. For intercountry comparisons, costs in THB can be converted into United States Dollar (USD) using the exchange rate of $\approx 30$ THB for 1 USD [11].

The primary aim of the study was to determine medical costs attributable to POAF among patients undergoing cardiac surgery. We first performed simple linear regression of log-transformed costs with preoperative patient characteristics as independent variables to identify potential confounders. Potential confounders were defined as those variables associated with the log-transformed cost with a $p$ value less than 0.2 on univariable analysis. The occurrence of POAF together with all potential confounders was forced to enter the multiple linear regression model, regardless of their statistically significant contribution to the final model. The heteroscedasticity of the regression residuals on the log scale was examined with White's test [12], and no significant heteroscedasticity was observed. Thus, the adjusted costs were retransformed back to their original scales with correction of retransformation bias using Duan's smearing estimator [13]. The attributable cost of POAF was determined by the mean difference of adjusted costs between patients with and without POAF.

Statistical analyses were performed using the Stata software. Unless otherwise indicated, the tested results were considered statistically significant with a $p$ value $<0.05$.

2.3. Ethical Issues. The study protocol was approved by the Institutional Review Board on Human Research at Naresuan University and the Human Research Committee at the study hospital prior to data collection.

\section{Results}

3.1. Patient Characteristics. The cohort consisted of 711 patients undergoing cardiac surgery during the study period. As shown in Table 1 , the mean age was $56.07 \pm 12.58$ years and more than half of the cohort were male. Hypertension was the most prevalent $(32.63 \%)$ comorbid condition. The majority $(68.50 \%)$ underwent isolated valve surgery, while $24.61 \%$ and $6.89 \%$ underwent isolated CABG and concomitant CABG with valve surgery, respectively.

Overall, POAF developed in 220 patients (30.94\%) during a median length of stay (LOS) of 10 days (interquartile range 3-17 days). Compared to those without POAF, patients with POAF were older (57.93 \pm 12.00 versus 55.24 \pm 12.58 ; $p=0.008)$ and had fewer diagnoses of atrial fibrillation prior to operation $(9.09 \%$ versus $15.07 \%$; $p=0.03)$, but more histories of chronic obstructive pulmonary disease, diabetes mellitus, dyslipidemia, hypertension, and ischemic heart diseases.

3.2. Resource Utilization. Patients with POAF spent 1 more day in hospital compared to those without POAF (median LOS, 11 days versus 10 days; $p<0.01$ ). The resource utilization among patients with and without POAF was compared, as shown in Table 2. On average, patients with POAF incurred higher unadjusted costs during hospitalization in every cost component $(p<0.001)$. The greatest difference was observed in costs associated with utilization of pharmacy products (19,210 THB (640 USD)) accounting for 35\% of the total mean difference.

3.3. The Attributable Cost of POAF. We adjusted the effects of potential confounders on the association between medical costs and the occurrence of POAF by developing a multivariable linear regression model. Because costs were heavily 
TABle 1: Preoperative characteristics of patients in the study.

\begin{tabular}{|c|c|c|c|c|}
\hline Characteristics & $\begin{array}{c}\text { All patients } \\
(N=711) n(\%)\end{array}$ & $\begin{array}{c}\text { With POAF } \\
(N=220) n(\%)\end{array}$ & $\begin{array}{l}\text { Without POAF } \\
(N=491) n(\%)\end{array}$ & $p$ value* \\
\hline Age (mean \pm SD) (years) & $56.07 \pm 12.58$ & $57.93 \pm 12.00$ & $55.24 \pm 12.58$ & 0.008 \\
\hline Male & $402(56.5)$ & $135(61.4)$ & $267(54.4)$ & 0.080 \\
\hline \multicolumn{5}{|l|}{ Comorbid conditions } \\
\hline Cerebrovascular disease & $2(0.3)$ & $1(0.5)$ & $1(0.2)$ & 0.560 \\
\hline Chronic kidney disease & $23(3.2)$ & $11(5.0)$ & $12(2.4)$ & 0.075 \\
\hline Congestive heart failure & $94(13.2)$ & $33(15.0)$ & $61(12.4)$ & 0.340 \\
\hline COPD & $23(3.2)$ & $12(5.5)$ & $11(2.2)$ & 0.025 \\
\hline Diabetes mellitus & $107(15.1)$ & $50(22.7)$ & $57(11.6)$ & $<0.001$ \\
\hline Dyslipidemia & $192(27.0)$ & $75(34.1)$ & $117(23.8)$ & 0.004 \\
\hline Hypertension & $232(32.6)$ & $92(41.8)$ & $140(28.5)$ & $<0.001$ \\
\hline Ischemic heart disease & $108(15.2)$ & $47(21.4)$ & $61(12.4)$ & 0.002 \\
\hline Preoperative atrial fibrillation & $94(13.2)$ & $20(9.1)$ & $74(15.1)$ & 0.030 \\
\hline Types of surgery & & & & 0.332 \\
\hline Isolated valve replacement & $487(68.5)$ & $144(65.5)$ & $343(69.9)$ & \\
\hline Isolated CABG & $175(24.6)$ & $62(28.2)$ & $113(23.0)$ & \\
\hline Valve replacement $+\mathrm{CABG}$ & $49(6.9)$ & $14(6.4)$ & $35(7.1)$ & \\
\hline
\end{tabular}

* Between patients with and without POAF using chi-square, except for age which was tested for the difference by independent $t$-test. COPD, chronic obstructive pulmonary disease; CABG, coronary artery bypass graft; POAF, postoperative atrial fibrillation.

TABLE 2: Medical costs according to service components in patients with and without POAF.

\begin{tabular}{|c|c|c|c|c|}
\hline Services & $\begin{array}{c}\text { With POAF } \\
(\mathrm{THB})\end{array}$ & $\begin{array}{c}\text { Without POAF } \\
\text { (THB) }\end{array}$ & $\begin{array}{c}\text { Mean difference } \\
(\mathrm{THB})\end{array}$ & $p$ value* \\
\hline Pharmacy & 43,328 & 24,118 & 19,210 & $<0.001$ \\
\hline Supplies and devices & 103,933 & 90,628 & 13,304 & $<0.001$ \\
\hline Room, board, and nursing & 26,771 & 18,593 & 8,178 & $<0.001$ \\
\hline Laboratory and testing & 23,948 & 17,709 & 6,238 & $<0.001$ \\
\hline Operations & 56,062 & 50,309 & 5,753 & $<0.001$ \\
\hline Others & 20,571 & 15,861 & 4,709 & $<0.001$ \\
\hline Total cost & 274,613 & 217,219 & 54,393 & $<0.001$ \\
\hline
\end{tabular}

${ }^{*}$ Independent $t$-test. POAF, postoperative atrial fibrillation; THB, Thai Baht.

right skewed, we log-transformed the total costs prior to regression modelling. Table 3 shows that, after adjusting for other variables, POAF still contributed significantly to the total cost $(\beta=0.21 ; 95 \%$ CI $0.15-0.27 ; p<0.001)$. In other words, a patient undergoing cardiac surgery who developed POAF had 23\% higher medical cost during hospitalization than a patient who did not develop POAF.

Table 4 shows that an occurrence of POAF incurred higher costs to patients undergoing either isolated valve replacement, CABG, or a combined procedure. The highest attributable cost was observed in patients undergoing a combined procedure (72,287 THB (2,410 USD) per patient).

\section{Discussion}

The results of the present study demonstrate that POAF is associated with higher medical cost independently of preoperative characteristics among patients undergoing cardiac surgery. The findings underscore a significant contribution of POAF to economic burden among patients who undergo cardiac surgery. To illustrate, about 10,000 patients undergo cardiac surgery in Thailand each year, and one-third of these patients will develop POAF. Given that POAF increases medical cost by 50,000 THB (1,667 USD) per patient according to our study, this will incur an additional cost of
Table 3: Predictors of total costs in multivariable linear regression model.

\begin{tabular}{lccc}
\hline Variables & $\beta$ & $95 \% \mathrm{CI}$ & $\begin{array}{c}p \\
\text { value }\end{array}$ \\
\hline Postoperative atrial fibrillation & 0.21 & $0.15-0.27$ & $<0.001$ \\
Age $>$ 60 years & -0.01 & $(-0.06)-0.05$ & 0.823 \\
Male sex & -0.01 & $(-0.06)-0.04$ & 0.731 \\
Preoperative atrial fibrillation & 0.08 & $(-0.00)-0.15$ & 0.057 \\
Chronic obstructive pulmonary & 0.14 & $(-0.00)-0.29$ & 0.058 \\
disease & & $0.07-0.22$ & $<0.001$ \\
Ischemic heart disease & 0.15 & $0.13-0.29$ & $<0.001$ \\
Congestive heart failure & 0.21 & $0.11-0.41$ & 0.001 \\
Chronic kidney disease & 0.26 & $-0.13)-(-0.01)$ & 0.027 \\
Dyslipidemia & -0.07 & $(-0.13)$ \\
\hline
\end{tabular}

170 million THB (5.7 million USD) annually. Thus, an effective intervention to prevent POAF or mitigate its severity if implemented would result in a significant reduction of health-care expenditure for the country.

The evidence that POAF prevention may save the cost of care in cardiac surgery can also be inferred from the costeffectiveness study of an intervention to prevent POAF. For example, Gillespie et al. [5] reported that use of betablockers for prophylaxis of POAF in cardiac surgery was associated with a significant reduction in the incidence of 
TABLe 4: Attributable costs of POAF according to types of surgery.

\begin{tabular}{|c|c|c|c|c|c|}
\hline Types of surgery & With POAF (THB) & Without POAF (THB) & Mean difference (THB) & $95 \% \mathrm{CI}$ & $p$ value* \\
\hline Isolated valve replacement & 268,740 & 220,979 & 47,761 & $39,809-55,712$ & $<0.001$ \\
\hline Isolated CABG & 266,500 & 215,635 & 50,865 & $37,233-64,496$ & $<0.001$ \\
\hline $\begin{array}{l}\text { Combined valve } \\
\text { replacement and CABG }\end{array}$ & 282,747 & 210,460 & 72,287 & $49,911-94,406$ & $<0.001$ \\
\hline All patients & 269,000 & 218,999 & 50,001 & $43,440-56,561$ & $<0.001$ \\
\hline
\end{tabular}

${ }^{*}$ Independent $t$-test. CABG, coronary artery bypass graft; POAF, postoperative atrial fibrillation; THB, Thai Baht.

POAF, hospital LOS, and total hospital cost. The results of our study agree with this finding and further emphasize beneficial effects of POAF prevention in cardiac surgery to Asian population.

Our data indicate that POAF was associated with more resource use from all service departments. In support of this observation, our results concurred with those reported by Hravnak et al., who found that POAF in patients undergoing isolated CABG resulted in more resource utilization, including laboratory test and pharmacy products [4]. The incremental cost was greatest in room services accounting for $26 \%$ of the total difference [4]. In our study, the major resource utilization driving the incremental cost of POAF was the pharmacy product utilization accounting for $35 \%$ of the total difference. This disparity is possibly explained by a variation in how service costs are established between hospitals, further emphasizing the confined application of economic data among various institutions and geographic locations. More importantly, both studies provided consistent evidence that the POAF incurs higher resource utilization in cardiac surgery patients.

We observed that patients with POAF stayed in hospital about 1 day longer than those without POAF. The difference in LOS would not totally explain the higher costs associated with POAF in this study. Consistent with our findings, previous studies reported that the LOS differed by approximately 1 day despite much higher costs observed among patients with POAF $[4,6]$. Other studies reported a longer incremental LOS, although the results varied widely from 3 to 8 days [5,7-10]. In a more recent study, the occurrence of POAF after a combined CABG and aortic valve replacement increased hospital LOS by 3 days with an incremental cost of 9,000 USD [14]. This discrepancy in LOS possibly reflects the difference in study population, practice pattern among institutions, and study designs. Despite the difference in LOS, all studies consistently observed an increase in medical costs associated with POAF [4-10].

Although the cost attributable to POAF was observed in all types of cardiac surgery, the highest incremental cost was associated with the combined procedure. Our results agree with those reported by Mahoney et al. [14], who found that the incremental cost associated with POAF was highest among patients undergoing a combined procedure. Additionally, LaPar et al. [15] reported that patients who underwent combined CABG and valve replacement accrued higher costs and longer LOS than did those who had an isolated procedure. Patients with combined cardiac surgery had higher rates of overall complication, and this may drive incremental cost of combined cardiac surgery [15]. We postulated that a greater incremental cost of POAF in patients undergoing a combined procedure was possibly due to more frequent complication related to POAF. From our data, medical costs were not much different among patients who underwent an isolated or a combined procedure and did not develop POAF (Table 4), suggesting that POAF itself likely incurred higher costs after a combined procedure. However, we cannot exclude the possibility of data imprecision due to a smaller number of patients undergoing a combined procedure. This finding should be further explored to gain additional insights on the economic burden of POAF in relation to distinct types of cardiac surgery and the impact of its prevention.

Some limitations of the present study merit discussion. First, this study utilized a cohort of patients to determine the attributable cost of POAF. Due to its retrospective and observational nature, the present study was subject to several factors confounding the association between cost and POAF. We tried to alleviate this problem by developing a regression model to adjust for preoperative characteristics; however, we acknowledge the presence of other unmeasured confounders. Previous studies revealed that preoperative together with intraoperative and postoperative characteristics explained variation in total costs of cardiac surgery better than preoperative factors alone [16-18]. Thus, the estimated cost in this study may not be totally adjusted for. Of note, most of the previous studies estimated the attributable cost of POAF by determining the difference in unadjusted costs between patients with and without POAF [4-8]. Such method would also subject to several potential confounders due to difference in baseline characteristics and disease severity of the participants. Second, the present study utilized secondary database as a data source, this may raise the question of result validity. Misclassification of POAF could have occurred if the POAF was perceived as insignificant and was not recorded into patient charts by care providers. The lack of ECG records from claims database prevented us from verifying the occurrence of POAF. This could lead to an underestimation of POAF incidence and false estimation of the POAF cost. Last, this study was conducted with data from a single institution, and this may limit generalizability of the result to other settings. However, the incidence of POAF and its effect on resource utilization observed in our study were well consistent with other studies [4-9]. We therefore believe that the results likely provide a valid and generalizable estimate of the attributable cost of POAF and could be used in assessing the 
cost-effectiveness of interventions to prevent an occurrence of POAF among patients undergoing cardiac surgery.

\section{Conclusions}

In a single-centre study in Thai population, POAF is consistently associated with an increase in medical costs among patients undergoing valve surgery, CABG, or the combination. Effective strategies to prevent POAF should be implemented to reduce its economic burden.

\section{Conflicts of Interest}

The authors declare no conflicts of interest in the present study.

\section{Authors' Contributions}

Pattamawan Kosuma carried out the study design, analysed data, and drafted the manuscript. Sitichok Wachirasrisirikul participated in the study design and drafted the manuscript. Arom Jedsadayanmata conceived the study, participated in the study design, and drafted the manuscript. All authors read and approved the final manuscript.

\section{Acknowledgments}

The authors acknowledge Naresuan University for the financial support of the present study.

\section{References}

[1] C. W. Hogue, L. L. Creswell, D. D. Gutterman, and L. A. Fleisher, "Epidemiology, mechanisms, and risks: American College of Chest Physicians guidelines for the prevention and management of postoperative atrial fibrillation after cardiac surgery," Chest, vol. 128, no. 2, pp. 9S-18S, 2005.

[2] W. H. Maisel, J. D. Rawn, and W. G. Stevenson, "Atrial fibrillation after cardiac surgery," Annals of Internal Medicine, vol. 135, no. 12, pp. 1061-1073, 2001.

[3] N. Echahidi, P. Pibarot, G. O’Hara, and P. Mathieu, "Mechanisms, prevention, and treatment of atrial fibrillation after cardiac surgery," Journal of the American College of Cardiology, vol. 51, no. 8, pp. 793-801, 2008.

[4] M. Hravnak, L. A. Hoffman, M. I. Saul, T. G. Zullo, and G. R. Whitman, "Resource utilization related to atrial fibrillation after coronary artery bypass grafting," American Journal of Critical Care, vol. 11, no. 3, pp. 228-238, 2002.

[5] E. L. Gillespie, C. M. White, J. Kluger, J. Sahni, R. Gallagher, and C. I. Coleman, "A hospital perspective on the costeffectiveness of $\beta$-blockade for prophylaxis of atrial fibrillation after cardiothoracic surgery," Clinical Therapeutics, vol. 27, no. 12, pp. 1963-1969, 2005.

[6] C. Rostagno, M. La mier, S. Gelsomino et al., "Atrial fibrillation after cardiac surgery: incidence, risk factors, and economic burden," Journal of Cardiothoracic and Vascular Anesthesia, vol. 24, no. 6, pp. 952-958, 2010.

[7] J. P. Mathew, R. Parks, J. S. Savino et al., "Atrial fibrillation following coronary artery bypass graft surgery: predictors, outcomes, and resource utilization," JAMA, vol. 276, no. 4, pp. 300-305, 1996.

[8] S. F. Aranki, D. P. Shaw, D. H. Adams et al., "Predictors of atrial fibrillation after coronary artery surgery: current trends and impact on hospital resources," Circulation, vol. 94, no. 3, pp. 390-397, 1996.

[9] G. H. Almassi, T. H. Wagner, B. Carr et al., "Postoperative atrial fibrillation impacts on costs and one-year clinical outcomes: the Veterans Affairs Randomized On/Off Bypass Trial," Annals of Thoracic Surgery, vol. 99, no. 1, pp. 109-114, 2015.

[10] G. H. Almassi, T. Schowalter, A. C. Nicolosi et al., "Atrial fibrillation after cardiac surgery: a major morbid event?," Annals of Surgery, vol. 226, no. 4, pp. 501-513, 1997.

[11] Bank of Thailand, "Foreign exchange rates," 2013, http://www. bot.or.th/thai/statistics/financialmarkets/exchangerate/_layouts/ application/exchangerate/exchangerate.aspx.

[12] H. White, "A heteroscedasticity-consistent covariance matrix estimator and a direct test for heteroscedasticity," Econometrica, vol. 48, no. 4, pp. 817-838, 1980.

[13] H. Duan, "Smearing estimate: a nonparametric retransformation method," Journal of the American Statistical Association, vol. 78, no. 383 , pp. 605-610, 1983.

[14] E. M. Mahoney, T. D. Thompson, E. Veledar, J. Williams, and W. S. Weintraub, "Cost-effectiveness of targeting patients undergoing cardiac surgery for therapy with intravenous amiodarone to prevent atrial fibrillation," Journal of the American College of Cardiology, vol. 40, pp. 737-745, 2002.

[15] D. J. LaPar, A. M. Speir, I. K. Crosby et al., "Postoperative atrial fibrillation significantly increases mortality, hospital readmission, and hospital costs," Annals of Thoracic Surgery, vol. 98, no. 2, pp. 527-533, 2014.

[16] E. Sokolovic, D. Schmidlin, E. R. Schmid et al., "Determinants of costs and resource utilization associated with open heart surgery," European Heart Journal, vol. 23, no. 7, pp. 574-578, 2002.

[17] C. M. Vassileva, J. Shabosky, T. Boley, S. Markwell, and S. Hazelrigg, "Cost analysis of isolated mitral valve surgery in the United States," Annals of Thoracic Surgery, vol. 94, no. 5, pp. 1429-1437, 2012.

[18] R. L. Osnabrugge, A. M. Speir, S. J. Head et al., "Prediction of costs and length of stay in coronary artery bypass grafting," Annals of Thoracic Surgery, vol. 98, no. 4, pp. 1286-1293, 2014. 


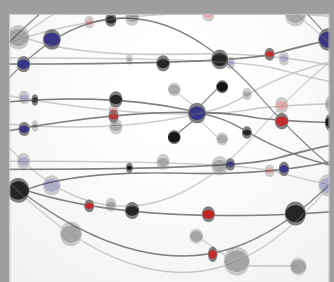

The Scientific World Journal
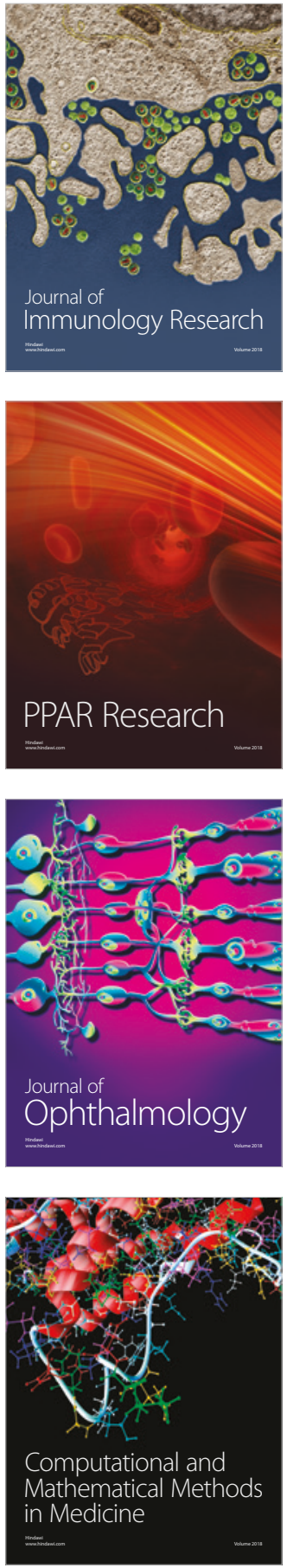

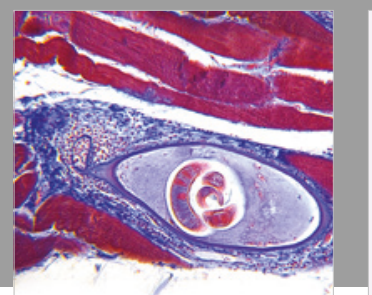

Gastroenterology Research and Practice

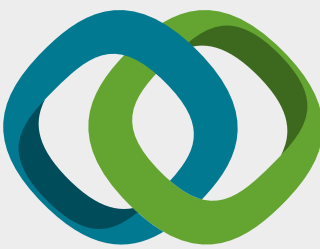

\section{Hindawi}

Submit your manuscripts at

www.hindawi.com
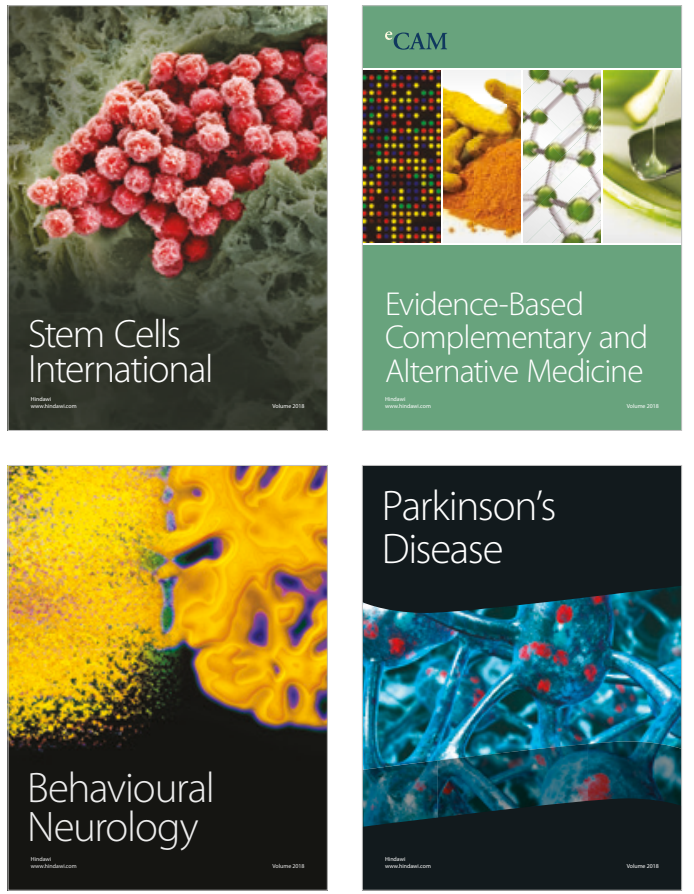

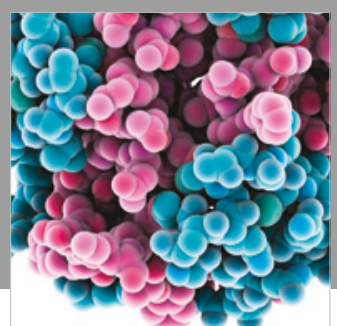

ournal of

Diabetes Research

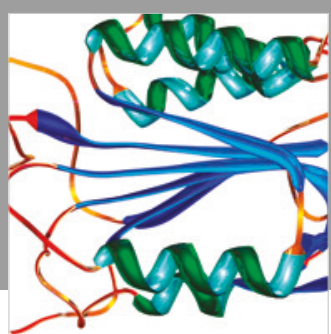

Disease Markers
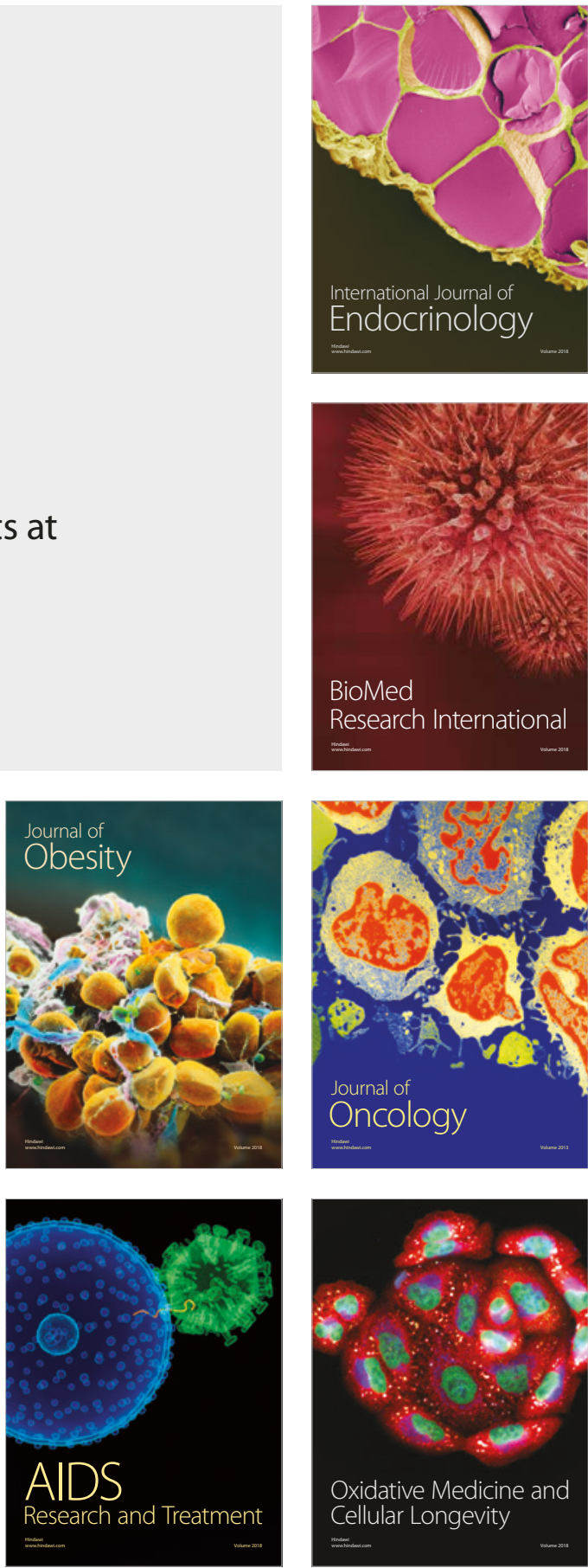